\title{
Combined clinical and genetic testing algorithm for cervical cancer diagnosis
}

\author{
Yu-Ligh Liou ${ }^{1,3,5 \dagger}$, Tao-Lan Zhang ${ }^{1 \dagger}$, Tian Yan², Ching-Tung Yeh ${ }^{5}$, Ya-Nan Kang ${ }^{2}$, Lanqin Cao ${ }^{2}$, Nayiyuan Wu', \\ Chi-Feng Chang ${ }^{5}$, Huei-Jen Wang ${ }^{5}$, Carolyn Yen ${ }^{5,6}$, Tang-Yuan Chu ${ }^{4,7,8}$, Yi Zhang ${ }^{2}$, Yu Zhang ${ }^{2 *}$ \\ and Honghao Zhou ${ }^{1,3^{*}}$
}

\begin{abstract}
Background: Opportunistic screening in hospitals is widely used to effectively reduce the incidence rate of cervical cancer in China and other developing countries. This study aimed to identify clinical risk factor algorithms that combine gynecologic examination and molecular testing (paired box gene 1 (PAX1) or zinc finger protein 582 (ZNF582) methylation or HPV16/18) results to improve diagnostic accuracy.

Methods: The delta Cp of methylated PAX1 and ZNF582 was obtained via quantitative methylation-specific PCR in a training set (57 CIN2 - and 43 cervical intraepithelial neoplasia $\geq$ grade 3 (CIN3+) women), and the individual and combination gene sensitivities and specificities were determined. The detection accuracy of three algorithms combining gynecologic findings and genetic test results was then compared in a randomized case-control study comprising 449 women referred for colposcopic examination by gynecologists in the outpatient department of Xiangya Hospital between November 2011 and March 2013.

Results: Significant association was observed between CIN3+ and methylated PAX1 or ZNF582 in combination with HPV16/18 (OR:15.52, 95 \% Cl:7.73-31.18). The sensitivities and specificities of methylated PAX1 or ZNF582 combined with HPV16/18 for CIN3+ women were 89.2 and $76.0 \%$, or 85.4 and $80.1 \%$, respectively. Of the three algorithms applied to cohort data and validated in the study, two indicated $100 \%$ sensitivity in detecting cervical cancer and a low rate of referrals for colposcopy.

Conclusions: These algorithms might contribute to precise and objective cervical cancer diagnostics in the outpatient departments of hospitals in countries with high mortality and low screening rates or areas with uneven resource distribution.
\end{abstract}

Keywords: Biomarkers, Algorithm, DNA methylation, HPV16/18, Cervical cancer, ZNF582, PAX1

\section{Background}

Cervical cancer is the fourth most common cancer that affects women worldwide. The use of the cytological test developed by George Papanicolaou (the Pap smear) in cervical cancer screening programs has led to a reduction in the incidence of cervical cancer in developed countries [1, 2]. However, most cases of cervical cancer are still associated with absent or deficient screening

\footnotetext{
* Correspondence: cszhangyu@126.com; hhzhou2003@163.com

${ }^{\dagger}$ Equal contributors

2Department of Obstetrics and Gynecology, Xiangya Hospital, Central South University, Hunan 410008, People's Republic of China

'Department of Clinical Pharmacology, Xiangya Hospital, Central South

University, Hunan 410078, People's Republic of China

Full list of author information is available at the end of the article
}

[3-6]. In China, cervical cancer remains the secondleading cause of death from cancer among females aged 15 to 44 years, and nearly 58,000 new cases and 20,000 deaths were documented in 2005 alone. In certain developed cities such as Beijing and Shanghai, the incidence of cervical cancer has dropped significantly because of the wide promotion of cervical cancer prevention and opportunistic screening in hospitals. Despite these advances, prevention awareness of cervical cancer is still inadequate in most areas of China because of its large population size $[7,8]$.

Oncogenic high-risk human papillomavirus (hrHPV) DNA testing is currently an appealing method for the molecular diagnosis of cervical cancer, as HPV plays an 
essential role in cervical carcinogenesis [9-12]. In China, the application of HPV testing has increased dramatically because of its consistency and reproducibility. Thus, HPV testing has increased to the level of an "HPV-heavy-burden of testing" in certain regions, with the prevalence varying significantly among different ages and regions [13]. Consequently, progressively more HPV-positive patients are in need of cytological or colposcopic examination in hospitals, which in turn highlights the deficiencies of the examination procedure and the overloading of the performing physicians [14]. HPV-positive assay results might also cause an adverse psychosocial impact on patients. P16/Ki67 dual staining is used as a tool for CIN2 triage; however, Wentzensen et al. reported that P16/Ki67 dual staining yielded a $78.9 \%$ positive rate for CIN2 and an $86.9 \%$ positive rate for CIN3/CIS/cancer but also exhibited a $41.1 \%$ positive rate for the CIN2- group [15]. Therefore, a more accurate method is required to reduce the high false-positive rate of hrHPV testing and to maintain the consistency and reproducibility of methods used in the outpatient departments of hospitals in China or other high-population countries.

One such screening possibility arises from the field of epigenetics [16]. Numerous investigations have reported that the gene-specific hypermethylation that occurs in the pre-invasive and invasive phases of cervical cancer might serve as a promising biomarker for early diagnosis $[17,18]$. Several studies examining paired boxed gene 1 (PAX1) and zinc finger protein 582 (ZNF582) have reported their potential utilization as highly sensitive biomarkers for detection of cervical intraepithelial neoplasia at grade 3 or higher (CIN3+) [19-26]. The PAX1 gene was found to be involved in the regulation of cell differentiation in head and neck cancer, and the ZNF582 gene is a protein-coding gene involved in gene expression [27, 28].

The aim of this study was to develop and verify effective clinical risk factor algorithms to increase the accuracy of diagnosis for cervical cancer based on existing validated candidate molecular tests and the highly weighted factor of clinical examination information from the subjects. Standardized quantitative methylation-specific PCR tests of methylated PAX1 gene $\left(P A X 1^{m}\right)$ and methylated ZNF582 gene $\left(Z N F 582^{m}\right)$ were performed on a full spectrum of cervical scrapings, with cutoff values selected from the training set, to determine the sensitivities and specificities for CIN3 + detection in the validation set. The gynecology examination results were also considered as a prominent risk factor in the algorithms for cervical cancer prediction. We propose that these algorithms would be useful in the outpatient departments of hospitals in China or other high-population countries.

\section{Case presentation}

\section{Patient recruitment and study design}

In accordance with the Declaration of Helsinki, all patients provided informed consent for their participation in the clinical study, whose protocol was approved by the Institutional Review Board of the Department of Clinical Pharmacology at Xiangya Hospital, Central South University, China. The clinical trial was registered in the Chinese Clinical Trial Registry (ChiCTR-DOD-14005446). Study inclusion criteria included females who were sexually active, not pregnant, had an intact uterus, and had no history of treatment for cervical intraepithelial neoplasia (CIN) or cervical cancer. Patients with a history of cancer related to the reproductive tract or therapy for cervical lesions or HPV vaccinations, in addition to those who had a current pregnancy, were excluded.

Female patients who had abnormal Pap smears, cervical inflammation, cervical erosion, or bleeding syndrome, or those who were suspected to have cervical cancer and were referred for colposcopic examination by the gynecologist in the outpatient department of the hospital, were included in this study. Patients were invited to the colposcopic examination room of the Department of Obstetrics and Gynecology to participate in a blinded study where testers were not provided clinical data. After patients signed an informed consent form, a standardized personal interview was given by experienced assistants, a case report form was filled, and every patient received a colposcopic examination and biopsy. The case report form included the inclusion and exclusion criteria, basic personal information, historic lifestyle and sickness records, histories of gynecologic examinations and findings, cytological results, and pathological results. The cytology results were classified according to the 2001 Bethesda System (TBS 2001) [29]. Colposcopy-directed biopsies were performed to provide histological results according to standard procedures in China. Biopsy specimens were histologically classified as normal, CIN1, CIN2, CIN3, cervical carcinoma in situ (CIS), squamous cell carcinoma (SCC), or adenocarcinoma (AC), according to the international criteria. The final diagnosis was based on the results of tissue-proven pathology. To ensure the quality of the diagnosis, two expert cytologists and two pathologists independently reviewed the cytology and histology slides, respectively. Standard guidelines for the management and treatment of cervical neoplasia were followed in all subjects [30]. All patient recruitment and clinical information collection processes were periodically monitored, and good clinical practice (GCP) guidelines were followed.

In the intervention arm of the randomized-controlled trial, data from the first 100 patients were used as a training set to build a prediction model that distinguished 
CIN3+ subjects from controls (Additional file 1: Table S1). The validation set was composed of the subsequent 466 patients $(17.6 \%$ of the total patients in the colposcopic room), with a mean age of 42.8 years (range, 27.577.8 years), enrolled from November 2011 to March 2013.

Twelve patients were excluded based on the exclusion criteria, and five were excluded because of poor quality of the DNA specimens. Thus, data from 449 patients were included in the final statistical analyses. The criteria used to determine positive and negative results for the tested methylated genes were based on the delta $\mathrm{Cp}$ of the training set (described in further detail below).

\section{Specimen collection and DNA preparation}

All liquid-based cytology samples were collected using CytoFast Solution (Hospitex Diagnostics SRL, Sesto Fiorentino, Italy). Residual cervical cells from cytological tests were used for HPV typing and methylation detection tests for the two genes. All specimens collected were assigned a number and delinked from patient clinical information until final data analysis. All molecular tests were performed at the Institute of Clinical Pharmacology, Hunan Key Laboratory of Pharmacogenetics, China, following good laboratory practice guidelines. The cells were centrifuged and stored in phosphate-buffered saline at $-20{ }^{\circ} \mathrm{C}$ from the day of collection. Genomic DNA (gDNA) was extracted from the collected cells using the QIAamp DNA Mini Kit (Qiagen GmbH, Hilden, Germany). A BioSpec-nano spectrophotometer (Shimadzu Corporation, Tokyo, Japan) was used to quantify the amount of extracted DNA.

\section{DNA methylation tests}

Quantitative methylation-specific PCR was performed using TaqMan-based technologies on the Lightcycler LC480 real-time PCR system (Roche Applied Sciences, Penzberg, Germany) with Cervi-P and Cervi-Z DNA detection kits (iStat Biomedical, Taipei, Taiwan). Briefly, $500 \mathrm{ng}$ of gDNA was subjected to bisulfite conversion using EZ DNA Methylation-Gold Kits (Zymo Research, Irvine, CA, USA). The methylation levels of the PAX1 and ZNF582 genes were then determined using the qPCR kits with internal controls according to the manufacturer's recommendations. The PCR reactions consisted of an initial incubation at $95{ }^{\circ} \mathrm{C}$ for $10 \mathrm{~min}$, followed by 50 cycles of denaturation at $95^{\circ} \mathrm{C}$ for $10 \mathrm{~s}$ and annealing and extension at $60{ }^{\circ} \mathrm{C}$ for $40 \mathrm{~s}$. Fluorescence data were collected during the annealing/extension step for $\mathrm{Cp}$ determination.

\section{HPV DNA amplification and genotyping}

The hrHPV-typing procedure was performed using a nested multiplex PCR assay that combined degenerate E6/E7 consensus primers and type-specific primers as previously described [31]. hrHPV type was determined after determining the size of the nested PCR amplification product.

\section{Algorithms for combining molecular tests and clinical gynecologic examination results}

To ascertain the clinical characteristics of the subjects, items of the case report form (Table 3) including gynecologic history were answered by patients, and the gynecologic examination results were recorded by the physicians for each patient in the study. The odds ratio for each clinical characteristic was determined by univariate analysis. The significantly associated factors were then used in a multivariate logistic regression analysis to select the variables based on goodness-of-fit analysis. Multiple regression analysis revealed that a gynecologic history of vaginal bleeding, grossly normal cervix (over $90 \%$ of the cervix had a uniform surface without warts or masses), and a finding of cervical bleeding during the gynecologic examination were associated with CIN3+ as shown in Table 3 (adjusted $P \leq 0.05$ ).

First, a logistic regression analysis based on molecular tests $\left(P A X 1^{m}\right.$ and/or $Z N F 582^{m}$, independently or both combined with HPV16/18) and key clinical characteristics (vaginal bleeding, grossly normal cervix, and finding of cervical bleeding) was used to discriminate between patients with CIN3+ and CIN2- cervical lesions. The methylated genes in the logistic regression formula for algorithms 1 to 3 were $P A X 1^{m}, Z N F 582^{m}$, and $P A X 1^{m}$ or ZNF582 ${ }^{m}$, respectively.

The logistic regression formula was as follows:

logistic score $=[W 1 *$ methylated gene OR $]+[W 2 * H P V 16 / 18 \mathrm{OR}]$ $+[W 3 *$ vaginal bleeding OR $]+[W 4 *$ grossly normal cervix OR $]+$ $[W 5 *$ contact bleeding $\mathrm{OR}]+C$ (constant number).

Second, the weight factor (W1-5; standardized regression coefficients) was calculated between the analytic results for the molecular tests and the key clinical characteristics. The weight factor value is a measure of how strongly each test influences the criterion variable (CIN3+ lesion). Each of the scores for the individual molecular tests and key clinical characteristics was entered into the logistic score as either "0" (negative) or "1" (positive). The results for the weight factor were calculated using the multivariate logistic regression.

For example:

Logistic score of algorithm $2:\left(2.334 * Z N F 582^{m} \mathrm{OR}\right)$

$+(1.485 *$ HPV $16 / 18$ OR $)+(0.529 *$ vaginal bleeding OR $)$

$+(-0.534 *$ grossly normal cervix OR $)$

$+(0.759 *$ contact bleeding OR $)-2.133$.

Each of the three algorithms differed in the weights and methylated genes used. Finally, the logistic score was transformed into a probability score. The probability 
score had a range of values from 0 to 1000 , which indicated the probability of CIN3+ for each algorithm [32].

The probability score was calculated as $\left[e^{\text {Logistic score }}\right] /$ $\left[1+e^{\text {Logistic score }}\right] * 1000$.

\section{Statistical analysis}

The cutoff values for each methylated gene were generated from the first 100 subjects, including 43 with CIN3 + results and 57 with CIN2- results. A cross-validated receiver operating characteristic (ROC) curve was generated, and the area under the ROC curve (AUC) was calculated for each detection method for CIN3+ lesions. The optimal cutoff value, i.e., the delta crossing point $(\triangle \mathrm{Cp})$, of each methylated gene was generated using the Youden index. The positive cutoff values for $P A X 1^{m}$ and $Z N F 582^{m}$ were determined as $\Delta \mathrm{Cp} \leq 9$ and $\Delta \mathrm{Cp} \leq 11$, respectively, from the first 100 subjects. SPSS software (version 16.0, Chicago, IL, USA) was used for all statistical analyses. Chi-squared and Fisher's exact tests were used to analyze the status of the methylated genes and HPV genotype in different combinations. Fisher's exact test is considered more accurate than the chi-squared test when the sample size is smaller than five. The sensitivity, specificity, and odds ratio (OR) with a $95 \%$ confidence interval (CI) for lesions of grade CIN3 or worse were calculated. All differences were considered two-sided and statistically significant at $P<0.05$. The algorithms were based on the multivariate logistic regression model.

\section{Discussion and evaluation}

Several previous studies have indicated that the analysis of $P A X 1^{m}$ and $Z N F 582^{m}$ in cervical cell scrapings and tissues holds great promise for detecting high-grade CIN lesions and cervical cancer $[19,22]$. However, these studies were conducted on selected populations such as within an outpatient referral case control study or following triage using cytology or hrHPV, wherein cytology was examined solely in LSIL patients. These study designs hampered the proper comparison of molecular test performance with cytology results, as patients with normal or partially abnormal results did not receive followup exams. The strengths of the present study lie in its incorporation of delinked random case-control study methods incorporating all histological results, including those of the normal group. This enabled the comparison of the results obtained with methylation markers with those of cytology or HPV genotyping for cervical cancer screening.

To our knowledge, this study is the first report to validate an opportunistic cervical cancer screening method that utilizes gynecologic examination findings, gynecologic history, and genetic biomarkers in combination to increase the accuracy of diagnoses under hospital outpatient conditions. In addition, we consider that this study also provides the first suggestion that that the majority of cervical cancer diagnoses in China or other developing countries should be obtained through opportunistic screening. Our results demonstrated that of the two genes used in the study, methylated ZNF582, with $76.6 \%$ sensitivity and $86.94 \%$ specificity for detecting cervical cancer, is a more promising biomarker. ZNF582 $2^{m}$ has been reported to function well in the triage of patients with equivocal liquid-based cytology results $[19,25]$. The second tested biomarker, $P A X 1^{\mathrm{m}}$, has also been reported as a useful biomarker for cervical cancer in the screening and triage of cytology and for the detection cervical adenocarcinoma. The results of this meta-analysis support the utility of $P A X 1^{\mathrm{m}}$ as an auxiliary biomarker in cervical cancer screening [33], as algorithm 1 demonstrated $89.87 \%$ sensitivity and $75.95 \%$ specificity for $P A X 1^{m}$ in combination with HPV16/18 and cytology testing, which is higher than the values obtained by testing any of these factors alone.

Both algorithms 2 and 3 showed a $100 \%$ detection rate in the cancer groups. We therefore recommend a new cervical cancer patient management strategy consisting of both algorithms 2 and 3 for use in opportunistic screening in hospitals. Patients who exhibit positive test results from algorithm 2 or 3 should then undergo a colposcopy examination or a biopsy. Upon obtaining negative results, patients should return for follow-up examinations at 6 months or 1 year. As a cancer management strategy, algorithm 2 might reduce the number of hrHPV-positive patients referred for colposcopy by $38.5 \%$, whereas algorithm 3 might reduce the referral number by $27.56 \%$; however, algorithm 3 also had an $83.33 \%$ positive rate for the histologic CIN3 category, which is $13.89 \%$ higher than that obtained with algorithm 2 .

In this study, the sensitivity and specificity of hrHPV testing and Pap smear tests applied individually for detecting CIN3+ were 98.1 and $46.1 \%$, and 69.0 and $90.7 \%$, respectively. However, in China, cytology and hrHPV diagnosis are time-consuming and impractical because of inaccurate results. Testing is performed in many hospitals that do not have sufficient medical resources to perform colposcopy and additional examinations, thus impeding proper diagnosis. Furthermore, colposcopy is invasive and causes anxiety in many patients. In comparison, the three proposed algorithms combine $P A X 1^{m}$ and/or ZNF582 ${ }^{m}$ with HPV16/18 testing and take into account gynecologic history/examination findings to enhance accuracy. These algorithms could improve the positive detection rate of CIN3+ lesions, with clinical observation and gene testing both proposed as follow-up measures. The use of these algorithms might thus greatly reduce the referral rate of hrHPV-positive patients and increase the accuracy of cytology in countries with limited resources for colposcopy. 
Table 1 Population and test characteristics by histologic category

\begin{tabular}{|c|c|c|c|c|c|c|c|c|}
\hline & \multirow[b]{2}{*}{ Cutoff } & \multicolumn{6}{|c|}{ Histological results } & \multirow[t]{2}{*}{ Total } \\
\hline & & Normal & CIN1 & $\mathrm{CIN} 2$ & CIN3 & $\mathrm{CIS}$ & SCC/AC & \\
\hline \multicolumn{9}{|l|}{ Number of subjects } \\
\hline$N(\%)$ & & $218(48.55)$ & $30(6.68)$ & $43(9.58)$ & $72(16.04)$ & $15(3.34)$ & $71(15.81)$ & $449(100)$ \\
\hline \multicolumn{9}{|l|}{ Age (years) } \\
\hline Mean \pm SD (range) & & $\begin{array}{l}41.2 \pm 10.6 \\
\text { (21.5 to } 77.8)\end{array}$ & $\begin{array}{r}47.0 \pm 9.6 \\
(29.0 \text { to } 66.2)\end{array}$ & $\begin{array}{r}39.2 \pm 11.1 \\
\text { (21.8 to } 64.6)\end{array}$ & $\begin{array}{r}37.3 \pm 5.1 \\
(23.7 \text { to } 63.0)\end{array}$ & $\begin{array}{r}45.1 \pm 8.7 \\
\text { (28.3 to } 65.7)\end{array}$ & $\begin{array}{r}42.9 \pm 4.5 \\
(32.5 \text { to } 74.3)\end{array}$ & $\begin{array}{l}42.8 \pm 10.3 \\
(21.5 \text { to } 77.8)\end{array}$ \\
\hline \multicolumn{9}{|l|}{ Cytology results } \\
\hline Normal (\%) & & $90(90.91)$ & $4(4.04)$ & $2(2.02)$ & $0(0.00)$ & $1(1.01)$ & $2(2.02)$ & $99(100)$ \\
\hline ASC-US (\%) & & $112(53.85)$ & $21(10.10)$ & $32(15.38)$ & $24(11.54)$ & $5(2.40)$ & $14(6.73)$ & $208(100)$ \\
\hline LSIL (\%) & & $1(16.67)$ & $2(33.33)$ & $0(0.00)$ & $3(50.00)$ & $0(0.00)$ & $0(0.00)$ & $6(100)$ \\
\hline ASC-H/AGC/HSIL+ (\%) & & $15(11.03)$ & $3(2.21)$ & $9(6.62)$ & $45(33.09)$ & $9(6.62)$ & $55(40.44)$ & $136(100)$ \\
\hline \multicolumn{9}{|c|}{ Detection modality or test used } \\
\hline hrHPV (\%) & & $100(45.87)$ & $18(60.00)$ & $39(90.70)$ & $71(98.61)$ & $15(100)$ & $69(97.18)$ & $312(69.49)$ \\
\hline HPV16/18 (\%) & & 19 (8.72) & $5(16.67)$ & $12(27.91)$ & $36(50.00)$ & $11(73.33)$ & $56(78.87)$ & 139 (30.96) \\
\hline$P A X 1^{m}(\%)$ & $\Delta C p \leqq 9.0$ & $34(15.60)$ & 4 (13.33) & $15(34.88)$ & $47(65.28)$ & $11(73.33)$ & $52(73.24)$ & $163(36.30)$ \\
\hline ZNF582 $2^{m}(\%)$ & $\Delta C p \leqq 11.0$ & $22(10.09)$ & $6(20.00)$ & $10(23.26)$ & $48(66.67)$ & $12(80.00)$ & 61 (85.92) & $159(35.41)$ \\
\hline
\end{tabular}


The present study has some potential limitations. For example, the subjects who were recruited were seen following referral for colposcopy examination and consisted of patients who had abnormal Pap smear results, inflammation syndrome, cervical erosion, bleeding syndrome, or suspected cervical cancer. In our cohort, $>90 \%$ of the patients had inflammation syndrome with positive hrHPV findings, which is not representative of the general population. In addition, many ASC-US patient samples were collected in the colposcopy room because most patients with obvious cervical cancer underwent biopsy immediately following abnormal Pap smear test results and clinical observation in the outpatient department. Other limitations include a small sample size and a lack of extensive and long-term follow-up information.

\section{Patient clinicopathological characteristics}

Cohort demographic characteristics, clinical information, and $P A X 1^{m}$ and $Z N F 582^{m}$ testing results are shown in Table 1. The cytology results of the cohort indicated that 99 were normal, 208 had atypical squamous cells of undetermined significance (ASC-US), 6 exhibited low-grade squamous intraepithelial lesion (LSIL), and 136 had atypical squamous cells for which HSIL/atypical glandular cell of undetermined significance/high-grade squamous intraepithelial lesion+ (ASC-H/ AGC/HSIL+) status could not be excluded. In total, colposcopy and biopsy revealed normal histology in 218 (48.55\%) patients, CIN1 in 30 (6.68 \%) patients, CIN2 in 43 (9.58\%) patients, CIN3 in $72(16.04 \%)$ patients, CIS in 15 patients (3.34 \%), and SCC/AC in 71 (15.81\%) patients. The positive rates of $P A X 1^{m}$ and $Z N F 582^{m}$ in the CIN3+ group were 69.6 and $77.6 \%$, respectively.

\section{Analytic sensitivity and specificity of the methylation analysis combined with HPV genotyping in the validation set}

Table 2 shows the results of the sensitivity, specificity, and accuracy for different molecular tests and their combinations for detection of CIN3+ lesions. The AUCs of HPV16/18, PAX1 ${ }^{m}$, and $Z N F 582^{m}$ in the validation set were $76.4,75.5$, and $81.8 \%$, respectively. The sensitivity and specificity of HPV16/18 for detecting CIN3+ lesions were 65.19 and $87.63 \%$, respectively, whereas $P A X 1^{m}$ showed $69.6 \%$ sensitivity (95 \% CI 62.1-76.3) and $81.8 \%$ specificity (95 \% CI 77.0-85.8), and ZNF582 ${ }^{m}$ exhibited $76.6 \%$ sensitivity (95 \% CI 69.4-83.0) and $86.9 \%$ specificity (95\% CI 82.6-90.3). We therefore analyzed PAX1 ${ }^{\mathrm{m}}$ and ZNF582 ${ }^{m}$ as reliable molecular tests in comparison to Pap smear results.

In the combined parallel testing of gene methylation with HPV genotyping, ZNF582 $2^{m}$ and $P A X 1^{m}$ combined with HPV16/18 results exhibited an AUC $>82 \%$ for detection of CIN3+. ZNF582 $2^{m}$ combined with HPV16/18 showed the best combination of high sensitivity (85.4\%) and high specificity $(81.1 \%)$, whereas $P A X 1^{m}$ combined with HPV16/18 had the highest sensitivity of $89.2 \%$ and a specificity of $76.0 \%$. The combination of both methylated genes, $Z N F 582^{m}$ and $P A X 1^{m}$, exhibited $85.4 \%$ sensitivity and $77.0 \%$ specificity.

\section{Algorithms combining gynecologic examination findings and genetic test results}

The crude ORs and corresponding $95 \%$ CIs for prediction of CIN3+ lesions in the study cohort were calculated. Univariate analyses revealed that age, number of pregnancies, contraceptive measures such as condom use, gynecologic history, and certain gynecologic examination findings were significantly $(P<0.05)$ associated with $\mathrm{CIN} 3+$, as shown in Table 3 . Patients who were over 50 years old (OR:2.97, $95 \%$ CI:1.13-7.8) and those from 30 to 50 years of age (OR:2.13, $95 \%$ CI:0.68-6.69) were found to have a higher risk of CIN3+ lesions compared to patients under 30 years of age. Patients who had given birth over three times or had a cervical mass finding on tumor examination were still considered to

Table 2 Performance of methylated genes and HPV tests for CIN3+ detection

\begin{tabular}{|c|c|c|c|c|c|}
\hline Tests & Sensitivity (\%) (95 \% Cl) & Specificity (\%) (95 \% Cl) & AUC (\%) $(95 \%$ Cl) & Odds ratio $(95 \% \mathrm{Cl})$ & $P$ value \\
\hline$\overline{P A X 1^{m}}$ & $69.62(62.05-76.26)$ & $81.79(76.95-85.80)$ & $75.5(70.8-80.6)$ & $10.29(6.55-16.16)$ & $<0.001$ \\
\hline ZNF582 $2^{m}$ & $76.58(69.40-82.51)$ & 86.94 (82.58-90.34) & $81.8(77.3-86.2)$ & $21.77(13.18-35.96)$ & $<0.001$ \\
\hline PAP & $68.99(61.40-75.68)$ & 90.72 (86.84-93.54) & $79.9(75.1-84.6)$ & 21.75 (12.93-36.59) & $<0.001$ \\
\hline hrHPV & 98.10 (94.57-99.35) & $46.05(40.41-51.79)$ & $72.1(67.5-76.7)$ & $44.10(13.75-141.45)$ & $<0.001^{*}$ \\
\hline HPV-16/18 & $65.19(57.48-72.18)$ & 87.63 (83.35-90.93) & $76.4(71.4-81.4)$ & $13.27(8.22-21.40)$ & $<0.001$ \\
\hline$P A X 1^{m}$ or hrHPV & 98.73 (95.50-99.65) & $42.61(37.06-48.35)$ & $70.7(66.0-75.3)$ & $57.92(14.09-238.17)$ & $<0.001^{*}$ \\
\hline$P A X 1^{m}$ or HPV-16/18 & $89.24(83.45-93.17)$ & 75.95 (70.72-80.50) & $82.6(78.5-86.6)$ & $26.19(14.80-46.33)$ & $<0.001$ \\
\hline$P A X 1^{m}$ or $Z N F 582^{m}$ & 85.44 (79.10-90.10) & 76.98 (71.81-81.44) & $81.2(76.9-85.5)$ & 19.62 (11.67-32.99) & $<0.001$ \\
\hline ZNF582 ${ }^{m}$ or hrHPV & $98.73(95.50-99.65)$ & $45.02(39.40-50.76)$ & $71.9(67.3-76.5)$ & 63.86 (15.53-262.56) & $<0.001^{*}$ \\
\hline ZNF582 $2^{m}$ or HPV-16/18 & 85.44 (79.10-90.10) & $81.10(76.21-85.18)$ & $83.3(79.2-87.4)$ & $25.19(14.82-42.82)$ & $<0.001$ \\
\hline
\end{tabular}

$P$ value determined by chi-squared test and *Fisher's exact test; odds ratio for CIN3+

$\mathrm{Cl}$ confidence interval, $\mathrm{HPV}$ human papillomavirus, hrHPV high-risk human papillomavirus, Gene ${ }^{m}$ methylated gene 
Table 3 Crude and adjust odds ratios (ORs) and corresponding $95 \%$ confidence intervals (Cls) for predictors of CIN3+ in cervical cancer

\begin{tabular}{|c|c|c|c|c|c|}
\hline \multirow[b]{2}{*}{ Variable } & \multicolumn{5}{|c|}{ Odds ratio $(95 \% \mathrm{Cl})$} \\
\hline & Number & Crude & $P$ value & Adjusted & $P$ value \\
\hline \multicolumn{6}{|c|}{ Age group } \\
\hline$<30$ & 52 & Reference & & Reference & \\
\hline $30-50$ & 319 & $3.93(1.72-9.00)$ & 0.001 & $2.97(1.13-7.80)$ & 0.027 \\
\hline$>50$ & 78 & $4.02(1.61-10.06)$ & 0.003 & $2.13(0.68-6.69)$ & 0.194 \\
\hline \multicolumn{6}{|c|}{ No. of pregnancies } \\
\hline 0 & 24 & Reference & & & \\
\hline $1 \sim 3$ & 232 & $2.68(0.89-8.11)$ & 0.081 & & \\
\hline$>3$ & 193 & $3.04(1.00-9.25)$ & 0.050 & & \\
\hline \multicolumn{6}{|c|}{ No. of births } \\
\hline 0 & 46 & Reference & & Reference & \\
\hline $1 \sim 3$ & 363 & $2.24(1.05-4.79)$ & 0.038 & $1.06(0.43-2.63)$ & 0.895 \\
\hline$>3$ & 40 & $4.54(1.75-11.83)$ & 0.002 & $1.71(0.53-5.56)$ & 0.370 \\
\hline \multicolumn{6}{|c|}{ Intrauterine device (IUD) usage } \\
\hline No & 215 & Reference & & & \\
\hline Yes & 234 & $1.41(0.95-2.08)$ & 0.087 & & \\
\hline \multicolumn{6}{|c|}{ Condom usage } \\
\hline No & 336 & Reference & & Reference & \\
\hline Yes & 113 & $0.59(0.37-0.94)$ & 0.027 & $0.78(0.46-1.31)$ & 0.349 \\
\hline \multicolumn{6}{|c|}{ Oral contraception usage } \\
\hline No & 391 & Reference & & & \\
\hline Yes & 58 & $0.81(0.45-1.46)$ & 0.478 & & \\
\hline \multicolumn{6}{|c|}{ Tubal ligation } \\
\hline No & 360 & Reference & & & \\
\hline Yes & 89 & $0.98(0.60-1.60)$ & 0.937 & & \\
\hline \multicolumn{6}{|c|}{ Gynecology history: cervical contact bleeding } \\
\hline No & 329 & Reference & & Reference & \\
\hline Yes & 120 & $1.61(1.05-2.47)$ & 0.030 & $1.11(0.68-1.80)$ & 0.673 \\
\hline \multicolumn{6}{|c|}{ Gynecology history: vaginal bleeding } \\
\hline No & 385 & Reference & & Reference & \\
\hline Yes & 64 & $3.77(2.18-6.54)$ & $<0.001$ & $2.95(1.62-5.39)$ & $<0.001$ \\
\hline \multicolumn{6}{|c|}{ Gynecology history: abnormal vaginal discharge } \\
\hline No & 439 & Reference & & & \\
\hline Yes & 10 & $1.87(0.53-6.56)$ & 0.329 & & \\
\hline \multicolumn{6}{|c|}{ Gynecologic examination: grossly normal cervix } \\
\hline No & 323 & Reference & & Reference & \\
\hline Yes & 126 & $0.40(0.25-0.65)$ & $<0.001$ & $0.51(0.30-0.85)$ & 0.011 \\
\hline \multicolumn{6}{|c|}{ Gynecologic examination : cervical erosion } \\
\hline No & 196 & Reference & & & \\
\hline Yes & 253 & $1.00(0.68-1.48)$ & 0.995 & & \\
\hline \multicolumn{6}{|c|}{ Gynecologic examination: cervical mass } \\
\hline No & 349 & Reference & & Reference & \\
\hline Yes & 100 & $2.01(1.28-3.15)$ & 0.003 & $1.37(0.83-2.25)$ & 0.220 \\
\hline
\end{tabular}


Table 3 Crude and adjust odds ratios (ORs) and corresponding $95 \%$ confidence intervals (Cls) for predictors of CIN3+ in cervical cancer (Continued)

\begin{tabular}{|c|c|c|c|c|c|}
\hline \multicolumn{6}{|c|}{ Gynecologic examination: ulcer } \\
\hline No & 446 & Reference & & & \\
\hline Yes & 3 & $3.72(0.33-41.33)$ & 0.285 & & \\
\hline \multicolumn{6}{|c|}{ Gynecologic examination: contact bleeding } \\
\hline No & 403 & Reference & & Reference & \\
\hline Yes & 46 & $2.95(1.58-5.50)$ & 0.001 & $1.94(0.99-3.83)$ & 0.055 \\
\hline
\end{tabular}
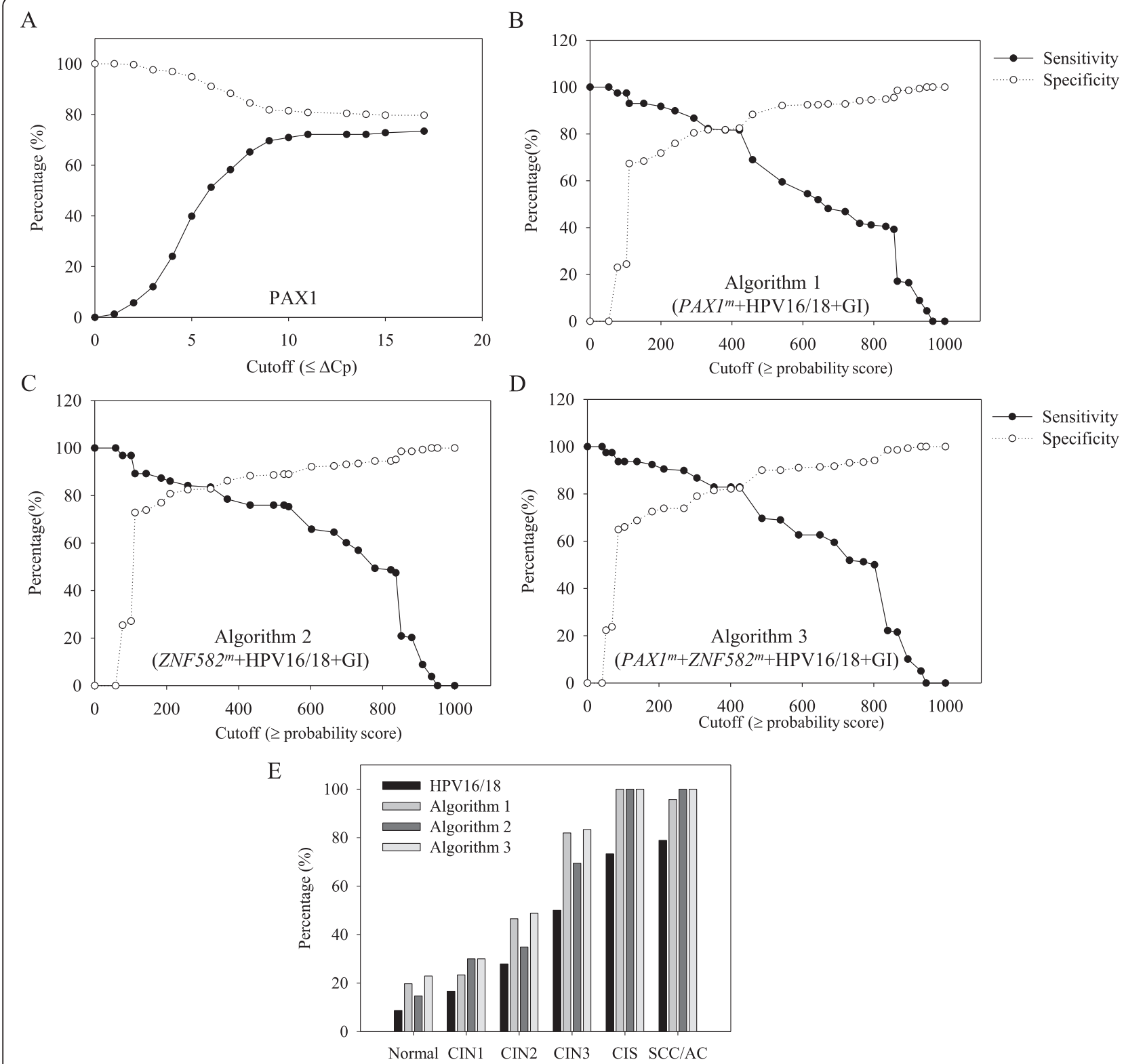

Fig. 1 Representative values plotted versus cutoff values in cervical cancer. a-d Illustrate the sensitivity and specificity of tests $\left(P A X I^{m}\right.$ and algorithms) at different cutoff values for detecting CIN3+ lesions. e Bar chart showing the positivity rate for algorithms in each histologic category when 220 was used as a cutoff value. Gl gynecologic information 
be at risk for cervical cancer. In the latter analysis, patients who had a gynecologic history of vaginal bleeding and examination findings of contact bleeding had an approximately 3.77- to 2.95-fold higher risk of CIN3+ lesions compared to those without these characteristics. In contrast, the gynecologic examination finding of a grossly normal cervix was negatively associated with $\mathrm{CIN} 3+$ lesions; furthermore, such patients exhibited a $50 \%$ lower risk of CIN3+ lesions compared to those with morphology changes.

Figure 1a shows the sensitivity and specificity of the $P A X 1^{m}$ gene only and the positive results determined at the $\Delta \mathrm{Cp} \leq$ cutoff value. Detection of the $P A X 1^{m}$ gene only had a limited sensitivity of approximately $80 \%$. Figure $1 b-d$ shows the range of cutoff values (probability score) of 0 to 1000 generated for algorithm 1 to 3, and the sensitivity and specificity of each algorithm were plotted with the different cutoff values (probability score). Finally, the optimal probability score was determined to be 220 in algorithms 1 to 3 by Youden's J statistic.

The bar chart in Fig. 1e shows the proportion of HPV $16 / 18$ and the detection rate for the other three algorithms with each histologic category. The positive detection rates for CIN3+ of the three algorithms were higher than those for HPV16/18 alone. All algorithms demonstrated over $86 \%$ sensitivity and $72 \%$ sensitivity for the detection of CIN3+ lesions (Table 4). Algorithm 1, which showed $89.87 \%$ sensitivity and $75.95 \%$ specificity, was $24.7 \%$ more sensitive and $11.7 \%$ less specific than HPV16/18 testing alone for detecting CIN3+ lesions, whereas algorithms 2 and 3 were 20.9 and $27.22 \%$ more sensitive and 6.9 and $15.12 \%$ less specific than HPV16/18, respectively.

For cancer detection, HPV16/18 had $77.90 \%$ sensitivity. Algorithm 1 showed $95.77 \%$ sensitivity, and algorithms 2 and 3 both exhibited $100 \%$ sensitivity. Algorithm 2 showed a $23.42 \%$ increase in sensitivity over ZNF582 testing alone for the histologic cancer (CIS/SCC/AC) category but showed only a $4.58 \%$ increase in sensitivity for detecting patients within the normal histologic category. Algorithm 2 also demonstrated a $22.10 \%$ increase in sensitivity for the histologic cancer (CIS/SCC/AC) category over HPV16/18 tests alone. Comparison of the hrHPV-positive rates obtained with the three algorithms with that of the CIN2- group indicated a decrease in the positivity rate obtained using algorithms $1-3$ to $29.9,34.71$, and $26.46 \%$, with a cancer detection rate of 95.77, 100, and $100 \%$, respectively.

Because of the large population, screening programs for cervical cancer are difficult to implement in China. However, the incidence of cervical cancer has decreased to a greater degree in urban areas than in rural areas because of the implementation of opportunistic screening in hospitals. Pap smear testing has been the major tool used for opportunistic screening in China over the past several decades. A survey of 202,231 patients in a retrospective opportunistic-screening study of 12 of the 3A hospitals in 2005 demonstrated that liquid-based cytology remained the major screening method in China [34], whereas hrHPV co-testing with cytology was utilized for approximately $11.7 \%$ of patients in the study. Accordingly, physicians face problems in delivering accurate diagnoses such as a low positive cytologic detection rate and a high false-positive rate of hrHPV detection, which result in insufficient treatment or over-treatment, respectively. To address these concerns, we suggest that combining gene testing with gross clinical history/examination findings in the outpatient departments of hospitals would provide a first step toward reducing the incidence of cervical cancer in China.

\section{Conclusions}

Algorithms that include molecular tests (methylated $P A X 1, Z N F 582$, and HPV16/18) in combination with clinical examination findings provide an effective method to increase the accuracy of diagnosis for cervical cancer. In this study, we established and validated algorithms that

Table 4 Sensitivity, specificity, and odds ratios of the three clinical risk factor algorithms for CIN3+ or CIS/SCC/AC detection

\begin{tabular}{|c|c|c|c|c|c|c|}
\hline Target genes & Cutoff & Sensitivity (\%) $(95 \%$ CI) & Specificity (\%) (95 \% Cl) & AUC (\%) $(95 \%$ Cl) & Odds ratio $(95 \% \mathrm{Cl})$ & $P$ value \\
\hline \multicolumn{7}{|l|}{ CIN3+ lesion } \\
\hline Algorithm 1 & 220 & 89.87 (84.18-93.67) & 75.95 (70.72-80.50) & 82.9 (78.9-86.9) & $28.02(15.65-50.17)$ & $<0.001$ \\
\hline Algorithm 2 & 220 & 86.08 (79.82-90.62) & 80.76 (75.84-84.87) & $83.4(79.3-87.5)$ & $25.94(15.17-44.36)$ & $<0.001$ \\
\hline Algorithm 3 & 220 & 92.41 (87.19-95.60) & $72.51(67.11-77.32)$ & $82.5(78.5-86.4)$ & 32.09 (16.88-61.00) & $<0.001$ \\
\hline \multicolumn{7}{|c|}{$\mathrm{CIS} / \mathrm{SCC} / \mathrm{AC}$} \\
\hline Algorithm 1 & 220 & $96.51(90.24-98.81)$ & $64.46(59.41-69.21)$ & $78.8(74.2-83.5)$ & 50.19 (15.55-161.98) & $<0.001^{*}$ \\
\hline Algorithm 2 & 220 & $100.0(94.87-100.0)$ & $70.80(65.92-75.24)$ & $84.0(80.4-87.6)$ & - & - \\
\hline Algorithm 3 & 220 & $100.0(94.87-100.0)$ & $61.43(56.33-66.30)$ & $79.5(75.3-83.7)$ & - & - \\
\hline
\end{tabular}

$P$ value determined by chi-squared test and *Fisher's exact test; odds ratio for CIN3+ or CIS/SCC/AC

$\mathrm{Cl}$ confidence interval, Gene ${ }^{m}$ methylated gene 
could be used as an objective screening method in the outpatient departments of hospitals to reduce the numbers of patients with cervical cancer. These algorithms might lead to the establishment of accurate, objective, non-morphological, and molecular-based test systems for cervical diagnosis in developing countries or countries where resources are not evenly distributed.

\section{Additional files}

Additional file 1: Table S1. Population and test characteristics by histologic category in training set. (PPTX $47 \mathrm{~kb}$ )

\section{Abbreviations}

AC, adenocarcinoma; AGC, atypical glandular cell of undetermined significance; ASC-H, atypical squamous cells cannot exclude high-grade squamous intraepithelial lesions; ASC-US, atypical squamous cells of undetermined significance; $A \cup C$, area under the curve; $\mathrm{Cl}$, confidence interval; CIN, cervical intraepithelial neoplasia; CIS, carcinoma in situ; GCP, good clinical practice; GLP, good laboratory practice; hrHPV, highrisk human papillomavirus; HSIL, high-grade squamous intraepithelial lesion; ORs, odds ratios; Pap smear, Papanicolaou smear; PAX1, paired box gene $1 ; P A X 1^{m}$, methylated $P A X 1$ gene; $R O C$, receiver operating characteristic; SCC, squamous cell carcinoma; ZNF582, zinc finger protein 582; ZNF582 ${ }^{m}$, methylated ZNF582 gene

\section{Acknowledgements}

We thank the women who participated in this study and their families. We also thank Chong-Zhen Oin, Pei-Yu Lv, Ying Zhang, Nayyuan Wu, Meng-Xue Ge, Rui-Jia Li, Chun Zhang, Juan Chen, Yue-Li Zhang, and Xing-Yu for the patient recruitment and clinical information collection.

\section{Authors' contributions}

$Y L L$ contributed to the writing of this manuscript and the coordination of the clinical trials and all molecular testing. YLL, YZ, CFC, TYC, and HHZ contributed to the conception, design, and final approval of the submitted version. YZ, YT, YNK, LC, and Yi Z contributed to the clinical data evaluation and selection. HJW, NW, CY, and TLZ contributed to the technical support for the gene testing results. YLL and CTY contributed to the data analysis. All authors read and approved the final manuscript.

\section{Competing interests}

iStat Biomedical Co., Ltd. supplied the testing kits used for the project. YLL is a Ph.D. student of the Department of Clinical Pharmacology, Xiangya Hospital, Central South University, China, and an employee of iStat Biomedical Co., Ltd., Taiwan. HJW, TYC, CY, and CFC are the employees of iStat Biomedical Co., Ltd. The other authors declare that they have no potential conflicts of interest.

\section{Ethics approval and consent to participate}

In accordance with the Declaration of Helsinki, all patients provided informed consent for their participation in the clinical study, whose protocol was approved by the Institutional Review Board of the Department of Clinical Pharmacology at Xiangya Hospital, Central South University, China. The clinical trial was registered in the Chinese Clinical Trial Registry (ChiCTRDOD-14005446)

\section{Author details}

${ }^{1}$ Department of Clinical Pharmacology, Xiangya Hospital, Central South University, Hunan 410078, People's Republic of China. ${ }^{2}$ Department of Obstetrics and Gynecology, Xiangya Hospital, Central South University, Hunan 410008, People's Republic of China. Institute of Clinical Pharmacology, Hunan Key Laboratory of Pharmacogenetics, Central South University, Changsha 410008, People's Republic of China. ${ }^{4}$ Department of Obstetrics and Gynecology, Buddhist Tzu Chi General Hospital, Hualien 97002, Taiwan. ${ }^{5}$ iStat Biomedical Co. Ltd., New Taipei City 22102, Taiwan. ${ }^{6}$ Department of Molecular and Cell Biology, University of California at
Berkeley, Berkeley, CA 94720-3200, USA. 'Institute of Medical Science, Tzu Chi University, Hualien 97002, Taiwan. ${ }^{8}$ Center for Cervical Cancer Prevention, Department of Research Buddhist, Tzu Chi General Hospital, Hualien 97002, Taiwan.

Received: 24 December 2015 Accepted: 29 May 2016

Published online: 10 June 2016

\section{References}

1. Arbyn M, Castelisagué X, de Sanjosé S, Bruni L, Saralya M, Bray F, et al. Worldwide burden of cervical cancer in 2008. Ann Oncol. 2011;22:2675-86.

2. Ferlay J, Shin HR, Bray F, Forman D, Mathers C, Parkin DM. Estimates of worldwide burden of cancer in 2008: GLOBOCAN 2008. Int J Cancer. 2010; 127:2893-917.

3. Cannistra SA, Niloff JM. Cancer of the uterine cervix. N Engl J Med. 1996;334:1030-8

4. Fahey MT, Irwig L, Macaskill P. Meta-analysis of Pap test accuracy. Am J Epidemiol. 1995;141:680-9.

5. Koliopoulos G, Arbyn M, Martin-Hirsch P, Kyrgiou M, Prendiville W, Paraskevaidis E. Diagnostic accuracy of human papillomavirus testing in primary cervical screening: a systematic review and meta-analysis of nonrandomized studies. Gynecol Oncol. 2007;104:232-46.

6. American College of Obstetricians and Gynecologists. ACOG practice bulletin. Clinical management guidelines for obstetrician-gynecologists. Number 61, April 2005. Human papillomavirus. Obstet Gynecol. 2005; 105:905-18.

7. Li J, Kang LN, Qiao YL. Review of the cervical cancer disease burden in mainland China. Asian Pac J Cancer Prev. 2011;12:1149-53.

8. Zhao P, Dai M, Chen W, Li N. Cancer trends in China. Jpn J Clin Oncol. 2010:40:281-5.

9. Baay MF, Weyler J, Vermorken JB. The value of human papillomavirus detection in primary cervical cancer screening. Eur J Gynaecol Oncol. 2004; 25:665-9.

10. Molijn A, Kleter B, Quint W, van Doorn L. Molecular diagnosis of human papillomavirus (HPV) infections. J Clin Virol. 2005;32:S43-51.

11. Baseman JG, Koutsky LA. The epidemiology of human papillomavirus infections. J Clin Virol. 2005;32:S16-24.

12. Mayrand MH, Duarte-Franco E, Rodrigues I, Walter SD, Hanley J, Ferenczy A, et al. Human papillomavirus DNA versus Papanicolaou screening tests for cervical cancer. N Engl J Med. 2007;357:1579-88.

13. Wang R, Guo XL, Wisman GB, Schuuring E, Wang WF, Zeng ZY, et al. Nationwide prevalence of human papillomavirus infection and viral genotype distribution in 37 cities in China. BMC Infect Dis. 2015;15:257.

14. Saslow D, Solomon D, Lawson HW, Killackey M, Kulasingam SL, Cain J, et al. American Cancer Society, American Society for Colposcopy and Cervical Pathology, and American Society for Clinical Pathology screening guidelines for the prevention and early detection of cervical cancer. Am J Clin Pathol. 2012;137:516-42.

15. Wentzensen N, Fetterman B, Castle PE, Schiffman M, Wood SN, Stiemerling E, et al. p16/Ki-67 dual stain cytology for detection of cervical precancer in HPV-positive women. J Natl Cancer Inst. 2015;107:djv257.

16. Esteller M. Epigenetics in cancer. N Engl J Med. 2008;358:1148-59.

17. Steenbergen RD, Snijders PJ, Heideman DA, Meijer CJ. Clinical implications of (epi) genetic changes in HPV-induced cervical precancerous lesions. Nat Rev Cancer. 2014;14:395-405.

18. Jones A, Lechner M, Fourkala EO, Kristeleit R, Widschwendter M. Emerging promise of epigenetics and DNA methylation for the diagnosis and management of women's cancers. Epigenomics. 2010;2:9-38.

19. Liou YL, Zhang Y, Liu Y, Cao L, Qin CZ, Zhang TL, et al. Comparison of HPV genotyping and methylated ZNF582 as triage for women with equivocal liquid-based cytology results. Clin Epigenetics. 2015;7:50

20. Chang CC, Huang RL, Wang HC, Liao YP, Yu MH, Lai HC. High methylation rate of LMX1A, NKX6-1, PAX1, PTPRR, SOX1, and ZNF582 genes in cervical adenocarcinoma. Int J Gynecol Cancer. 2014;24:201-9.

21. Kan YY, Liou YL, Wang HJ, Chen CY, Sung LC, Chang CF, et al. PAX1 methylation as a potential biomarker for cervical cancer screening. Int J Gynecol Cancer. 2014;24:928-34.

22. Lai HH, Qu YC, Chen TC, Huang HJ, Cheng YM, Chen CH, et al. PAX1/SOX1 DNA methylation and cervical neoplasia detection: a Taiwanese gynecologic oncology group (TGOG) study. Cancer Med. 2014;3:1062-74. 
23. Chao TK, Ke FY, Liao YP, Wang HC, Yu CP, Lai HC. Triage of cervical cytological diagnoses of atypical squamous cells by DNA methylation of paired boxed gene 1 (PAX1). Diagn Cytopathol. 2011;41:41-6.

24. Lin H, Chen TC, Chang TC, Cheng YM, Chen CH, Chu TY, et al. Methylated ZNF582 gene as a marker for triage of women with Pap smear reporting low grade squamous intraepithelial lesions - a Taiwanese gynecologic oncology group (TGOG) study. Gynecol Oncol. 2014;135:64-8.

25. Lai HC, Lin YW, Huang RL, Chung MT, Wang HC, Liao YP, et al. Quantitative DNA methylation analysis detects cervical intraepithelial neoplasms type 3 and worse. Cancer. 2010;116:4266-74.

26. Huang RL, Chang CC, Su PH, Chen YC, Liao YP, Wang HC, et al. Methylomic analysis identifies frequent DNA methylation of zinc finger protein 582 (ZNF582) in cervical neoplasms. PLoS One. 2012;7:e41060

27. Guerrero-Preston R, Michailidi C, Marchionni L, Pickering CR, Frederick MJ, Myers JM, et al. Key tumor suppressor genes inactivated by "greater promoter" methylation and somatic mutations in head and neck cancer. Epigenetics. 2014;9:1031-46.

28. Bhat S, Kabekkodu SP, Noronha A, Satyamoorthy K. Biological implications and therapeutic significance and DNA methylation regulated genes in cervical cancer. Biochimie. 2016;121:298-311.

29. Solomon D, Davey D, Kurman R, Moriarty A, O'Connor D, Prey M, et al. The 2001 Bethesda System: terminology for reporting results of cervical cytology. JAMA. 2002;287:2114-9.

30. Ngan HY, Garland SM, Bhatla N, Pagliusi SR, Chan KK, Cheung AN, et al. Asia Oceania guidelines for the implementation of programs for cervical cancer prevention and control. J Cancer Epidemiol. 2011:2011:794861.

31. Lin H, Moh JS, Ou YC, Shen SY, Tsai YM, ChangChien CC, et al. A simple method for the detection and genotyping of high-risk human papillomavirus using seminested polymerase chain reaction and reverse hybridization. Gynecol Oncol. 2005;96:84-91.

32. Imperiale TF, Ransohoff DF, Itzkowitz SH, Levin TR, Lavin P, Lidgard GP, et al. Multitarget stool DNA testing for colorectal-cancer screening. N Engl J Med. 2014:370:1287-97.

33. Nikolaidis C, Nena E, Panagopoulou M, Balgkouranidou I, Karaglani M, Chatzaki E, et al. PAX1 methylation as an auxiliary biomarker for cervical cancer screening: a meta-analysis. Cancer Epidemiol. 2015;39:682-6.

34. Tu Z, Xu A, Bian M, et al. Opportunistic screening for cervical cancer in 12 hospitals in China. J Practical Obstet Gynaecol. 2009;5:278-81.

\section{Submit your next manuscript to BioMed Central and we will help you at every step:}

- We accept pre-submission inquiries

- Our selector tool helps you to find the most relevant journal

- We provide round the clock customer support

- Convenient online submission

- Thorough peer review

- Inclusion in PubMed and all major indexing services

- Maximum visibility for your research

Submit your manuscript at www.biomedcentral.com/submit

) Biomed Central 